\title{
Enhanced light-matter interactions in dielectric nanostructures via machine-learning approach
}

\author{
Lei $\mathrm{Xu},{ }^{\mathrm{a}, \mathrm{b}}$ Mohsen Rahmani, ${ }^{\mathrm{b}, \mathrm{c}, \mathrm{d}, \boldsymbol{}}$ Yixuan Ma, ${ }^{\mathrm{a}}$ Daria A. Smirnova, ${ }^{\mathrm{c}}$ Khosro Zangeneh Kamali, ${ }^{\mathrm{c}, \mathrm{d}}$ Fu Deng, ${ }^{\mathrm{a}}$ \\ Yan Kei Chiang, ${ }^{a}$ Lujun Huang, ${ }^{a}$ Haoyang Zhang, ${ }^{e}$ Stephen Gould, ${ }^{f}$ Dragomir N. Neshev, ${ }^{c, d}$ and \\ Andrey E. Miroshnichenko, \\ aniversity of New South Wales, School of Engineering and Information Technology, Canberra, Australia \\ ${ }^{b}$ Nottingham Trent University, School of Science \& Technology, Department of Engineering, Advanced Optics and Photonics Laboratory, \\ Nottingham, United Kingdom \\ ${ }^{c}$ Australian National University, Research School of Physics, Nonlinear Physics Centre, Canberra, Australia \\ ${ }^{d}$ Australian National University, Research School of Physics, ARC Centre of Excellence for Transformative Meta-Optical Systems (TMOS), \\ Canberra, Australia \\ ${ }^{e}$ Queensland University of Technology, School of Electrical Engineering and Computer Science, Brisbane, Queensland, Australia \\ ${ }^{\dagger}$ Australian National University, College of Engineering and Computer Science, Canberra, Australia
}

\begin{abstract}
A key concept underlying the specific functionalities of metasurfaces is the use of constituent components to shape the wavefront of the light on demand. Metasurfaces are versatile, novel platforms for manipulating the scattering, color, phase, or intensity of light. Currently, one of the typical approaches for designing a metasurface is to optimize one or two variables among a vast number of fixed parameters, such as various materials' properties and coupling effects, as well as the geometrical parameters. Ideally, this would require multidimensional space optimization through direct numerical simulations. Recently, an alternative, popular approach allows for reducing the computational cost significantly based on a deep-learning-assisted method. We utilize a deep-learning approach for obtaining high-quality factor (high- $Q$ ) resonances with desired characteristics, such as linewidth, amplitude, and spectral position. We exploit such high- $Q$ resonances for enhanced light-matter interaction in nonlinear optical metasurfaces and optomechanical vibrations, simultaneously. We demonstrate that optimized metasurfaces achieve up to 400 -fold enhancement of the third-harmonic generation; at the same time, they also contribute to 100 -fold enhancement of the amplitude of optomechanical vibrations. This approach can be further used to realize structures with unconventional scattering responses.

Keywords: machine learning; dielectric nanostructures; Fano resonance; third-harmonic generation; optoacoustics.
\end{abstract}

Received Mar. 15, 2020; accepted for publication Apr. 13, 2020; published online Apr. 29, 2020.

(C) The Authors. Published by SPIE and CLP under a Creative Commons Attribution 4.0 Unported License. Distribution or reproduction of this work in whole or in part requires full attribution of the original publication, including its DOI.

[DOI: 10.1117/1.AP.2.2.026003]

\section{Introduction}

Metasurfaces are thin and flat arrays of subwavelength nanoparticles, enabling control over the polarization, phase, amplitude, and dispersion of light. ${ }^{1}$ They can be used for light emission, detection, modulation, control, and/or amplification at the nanoscale. In recent years, metasurfaces have been a subject of ongoing intense studies as their optical properties can be adapted to a diverse set of applications, including superlenses, tunable images, holograms, etc. ${ }^{1-5} \mathrm{High}-$ refractive-index dielectric metasurfaces provide a powerful platform for controlling light that

*Address all correspondence to Mohsen Rahmani, E-mail: mohsen.rahmani@anu .edu.au; Andrey E. Miroshnichenko, E-mail: andrey.miroshnichenko@unsw.edu.au can go beyond plasmonics as they cause negligible losses as compared with plasmonic metasurfaces. Dielectric materials offer the unique ability to efficiently manipulate light at the nanoscale based on the simultaneous excitation and control over the optically induced electric and magnetic Mie-type resonances. Resonant dielectric metasurfaces with high-quality factor (high- $Q$ ) resonances, in particular, are of significant interest due to their ability to strongly enhance the electromagnetic near-fields and boost the light-matter interactions at the nanoscale. In other words, they allow for enhancing the response of metasurfaces to an external electromagnetic field at a particular frequency. Moreover, high- $Q$ metasurfaces can increase the storage time of photons and, thus, light-matter interactions within the subwavelength resonators. This will facilitate 
various nanophotonics applications, such as enhanced nonlinear photon generation, optical sensing, optoacoustic vibrations, and narrowband filtering. In the last decade, high- $Q$ metasurfaces were mainly associated with Fano resonances (FRs) featuring asymmetric spectral line profiles. ${ }^{6-10}$ In FRs, the asymmetry originates from a close interaction of a discrete (localized) state with a continuum of propagation modes. ${ }^{11-13}$ Some examples are trapped modes in arrays of dielectric nanodisks with asymmetric holes,${ }^{14-16}$ ring and disk cavities, ${ }^{17}$ Dolmen structures, ${ }^{18,19}$ and aggregated nanoparticles. ${ }^{20-23}$ Recently, different approaches for generating high- $Q$ resonances have been based on the boundstate-in-the-continuum (BIC), i.e., a localized state with zero linewidth that is embedded in the continuum. ${ }^{15,24-32}$ Indeed, optical BICs provide a unique opportunity to manipulate the light-matter interaction within the radiative continuum because of their ultrahigh- $Q$ origin and associated giant enhancement of the electromagnetic near-field. ${ }^{33}$

By contrast, designing metasurfaces with high- $Q$ resonances is usually achieved via continuous tuning of parameters, with limited control on the linewidth, amplitude, and spectral positions. Currently, one of the typical approaches for designing metasurfaces with the desired resonance is based on direct optimization of one or two parameters via brute-force simulations. ${ }^{34-44}$ This is a time-consuming task accompanied by a random success on the output parameters of the desired resonances. ${ }^{45}$ Recently, deeplearning approaches, based on artificial neural networks (ANNs), have emerged as a revolutionary and robust methodology in nanophotonics. ${ }^{46-60}$ Indeed, applying deep learning algorithms to the nanophotonic inverse design can introduce remarkable design flexibility that can exceed that of the conventional methods. ${ }^{61}$ The inverse-design approach is based on a training process that enables fast prediction of complex optical properties of nanostructures with intricate architectures.

As a (nonunique) example, we have targeted toroidal dipoles (TDs) due to their promising applications in the formation of anapole states and electromagnetic energy localization. We investigate the nonradiating TD supported by two parallel silicon bars as the building blocks of the metasurface. The reason for choosing such a geometry is that it allows for a reasonable number of parameters to be optimized as a proof of concept. The parameters include the length and width of the bars, as well as the gap between them. At the same time, this TD corresponds to the symmetry-protected BIC. Subsequently, with this TDBIC model, we demonstrate the deep-learning-assisted inverse design of arbitrary high- $Q$ resonances with different line widths, amplitudes, and spectral positions. We employ a multilayer perceptron (MLP) variant of the ANN as our model. ${ }^{62-64}$ An MLP consists of multiple layers of perceptrons, including an input layer and an output layer with several hidden layers. In this work, each artificial neuron in one layer connects with a certain weight to every neuron in the following layers that are adapted during the learning state. Once learned, the weight values remain fixed, and the model can be used to infer the target metasurface design parameters. The hidden layers establish a nonlinear mapping between the input and output through training from the given dataset, which is then able to predict the response of the system or inversely determine the design parameters for the desired performance.

The proposed deep-learning-assisted inverse-design approach provides a platform to design metasurfaces for more than one application. In this paper, we employ machine learning to obtain a bifunctional metasurface dealing with photons and phonons, simultaneously. ${ }^{65-74}$ Photon-photon conversions, so-called nonlinear nanophotonics, is at the heart of modern macroscopic optics, including lasers, sensors, imaging, and information technology. By contrast, photon-phonon conversions are the state-of-the-art solution for precision mass sensors, micromanipulation, and sensing biochemical materials, with transformative implications in the fields of health and security. A combined photon-phonon conversion can be used for nonionizing and noninvasive imaging. ${ }^{73}$ Here, using the deep-learningassisted inverse approach, we design and fabricate a single optoacoustic metasurface that enhances third-harmonic generation (THG) intensity up to 400 times and acoustic mode excitations up to 100 times, concurrently, all through a designed high- $Q$ resonance associated with a strong electric near-field enhancement. The inverse-design approach proposed in this paper is extendable to other characteristics and applications of metasurfaces and significantly circumvents the time-consuming, case-by-case numerical models in conventional electromagnetic nanostructure designs.

\section{Results and Discussions}

To obtain the initial high- $Q$ resonances, we have defined the building blocks of metasurfaces to be two identical silicon nanobars with width $w$, length $L$, and the offset $x_{0}$, which is the distance between the center of the two bars fabricated on a glass substrate, as shown in the top panel of Fig. 1(a). It is worth mentioning that a large variety of other geometries, for generating high- $Q$ resonances have been demonstrated. ${ }^{15,24-31,35,39}$ However, discussions on advantages and disadvantages of various geometries are beyond the scope of the current study. Instead, we concentrate on customizing the generated high- $Q$ resonances. The thickness of bars is fixed at $150 \mathrm{~nm}$, and the periodicity of unit cells is fixed at $D=900 \mathrm{~nm}$ in both the $x$ and $y$ directions. As can be seen in the bottom panel of Fig. 1(a), this structure supports a strong FR in the transmission spectrum when the incident light is polarized along the $y$ axis. This FR is formed by the interference and coupling between a "bright" electric dipole (ED) resonance $p_{y}$ and a "dark" TD mode $T_{y}$. Figure 1(b) shows the corresponding spherical multipolar decomposition of the metasurface. As can be seen, the optical response is dominated by ED excitation with a small contribution from the magnetic quadrupole (MQ) resonance. Such a pronounced ED feature was further investigated by performing the Cartesian multipolar analysis [Fig. 1(c)]. It is worth noting that the ED response is mainly due to the strong excitation of TD mode $T_{y}$ with an inplane ED mode $p_{y}$, which is polarized along the same direction as the optical pump.

Interestingly, due to the $C_{2}$ symmetry of our subdiffractive system, the TD and MQ do not contribute to the far-field radiation along the $z$ direction. The far-field optical response is dominated by the ED mode $p_{y}$. The nonradiating TD mode is a symmetry-protected $\mathrm{BIC}$, where the ED mode $p_{y}$ plays a role in opening a leaky channel and transforming this ideal BIC into a quasi-BIC with a finite $Q$-factor. In our previous work, ${ }^{15}$ we studied the formation of BIC enabled by a magnetic dipole (MD) resonance in Si disk-with-hole metasurfaces, where geometrical asymmetry was introduced to open a leaky channel. In contrast, here we show that the leaky channel, i.e., the excitation of ED mode $p_{y}$, can be formed directly by properly choosing the structural dimensions of the symmetric nanobars. Owing to the nonradiating nature of the dominant resonance-TD mode, a clear enhancement of the stored electric energy inside the 
(a)

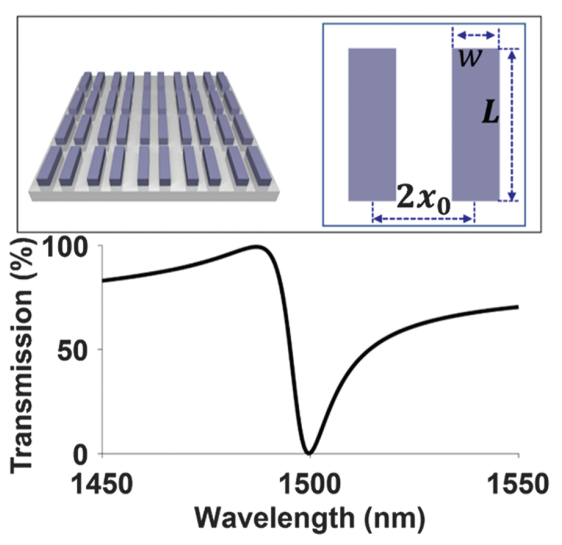

(c)

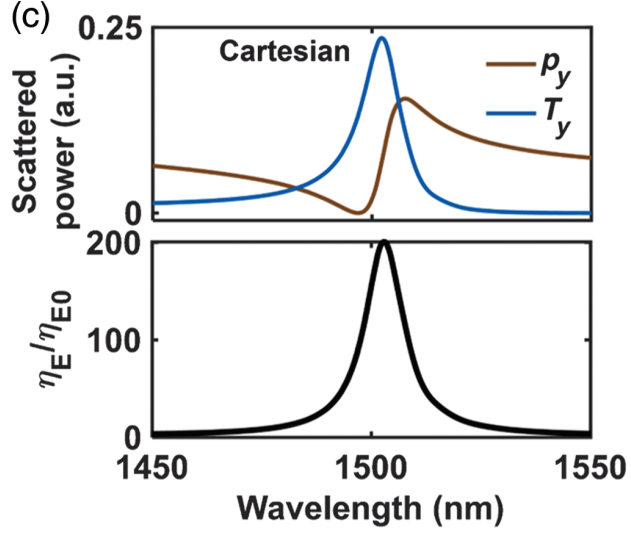

(b)

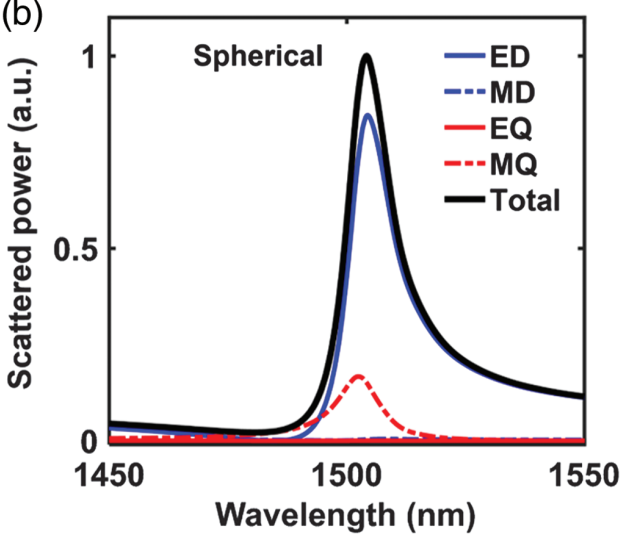

(d)

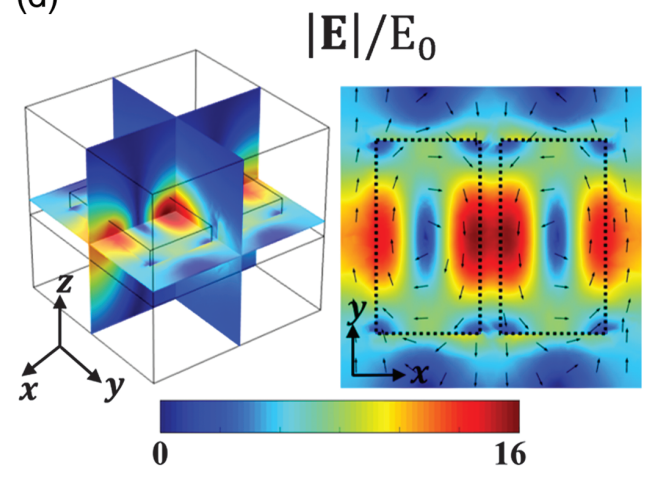

Fig. 1 (a) Top: (top left) Schematics of the silicon nanobars metasurface and (top right) its unit cell. Bottom: Calculated transmission spectrum of the metasurface with structural parameters $w=316 \mathrm{~nm}, L=580 \mathrm{~nm}, x_{0}=189 \mathrm{~nm}$. (b) Spherical multipolar structure of the metasurface. (c) Top: Cartesian ED and TD modes excitations. Bottom: The electric energy enhancement $\eta_{E} / \eta_{E_{0}}$. It is defined as the electric energy inside the two nanobars normalized by the electric energy within the same volume of the nanobars for the pump field. (d) Electric near-field distributions at the resonance. Left: 3-D view. Right: top view.

nanobars is observed, as shown in the bottom of Fig. 1(c). Figure 1(d) gives the calculated electric near-field distributions. A pronounced poloidal current distribution can be observed from the two nanobars, indicating strong TD excitation. The small portion of MQ excitation shown in Fig. 1(d) is due to the uncompensated circulating magnetic field in the flat geometry, formed by two antiparallel MD moments at the nodes of the poloidal current distribution. A comparison between the electric near-fields between the $y$-polarized pump and $x$-polarized pump incidence can be seen in Fig. S1 of the Supplemental Material. We observed significant near-field enhancement inside the nanostructure for the $y$-polarized pump incidence as compared with the case for the $x$-polarized pump incidence. A further investigation on the band structure and the corresponding mode profiles can be seen in Figs. S2 and S3 of Section I in the Supplemental Material.

As mentioned earlier, achieving scalable metasurfaces with several interdependent characteristics, including quality factor and spectral position, is the main target of this paper. There is a significant demand in the photonics community to achieve both conditions rather than solely obtaining a high- $Q$ resonance. In this respect, we employ the deep-learning approach to inversely design our metasurfaces while simultaneously controlling and optimizing on $Q$-factor, amplitude, and spectral position.
For this task, we use the open-source neural-network library Keras $^{75}$ written in Python to implement our method.

For the inverse design of nanophotonic structures using a deep-learning approach, one challenge is that the same far-field electromagnetic response can correspond to different designs, i.e., several different structures can give the same responses. This nonuniqueness of the response-to-design mapping will induce conflicting examples within the training set and may prevent convergence. To avoid this issue, we use the tandem network (TN) approach, ${ }^{46,56}$ as shown in Fig. 2. The TN architecture consists of an inverse-design network connected to a forward model network. The forward network learns the mapping from the structural parameters to the optical responses and is trained separately first. After the forward network is trained, it is placed after the inverse-design model network, and its network weights remain fixed during the training of the inversedesign model network. The inverse-design network learns a mapping from the optical responses to the structural parameters. When training the inverse-design network, its weights are updated to minimize the loss objective: $J=\frac{1}{n} \sum_{i}\left(r_{i}-o_{i}\right)^{2}$ with $r_{i}$ and $o_{i}$ being the values predicted by the neural network and the ground truth of the response (forward model network) or the structural parameters (inverse-design model network). Trained in this way, the inverse-design network is not constrained to 


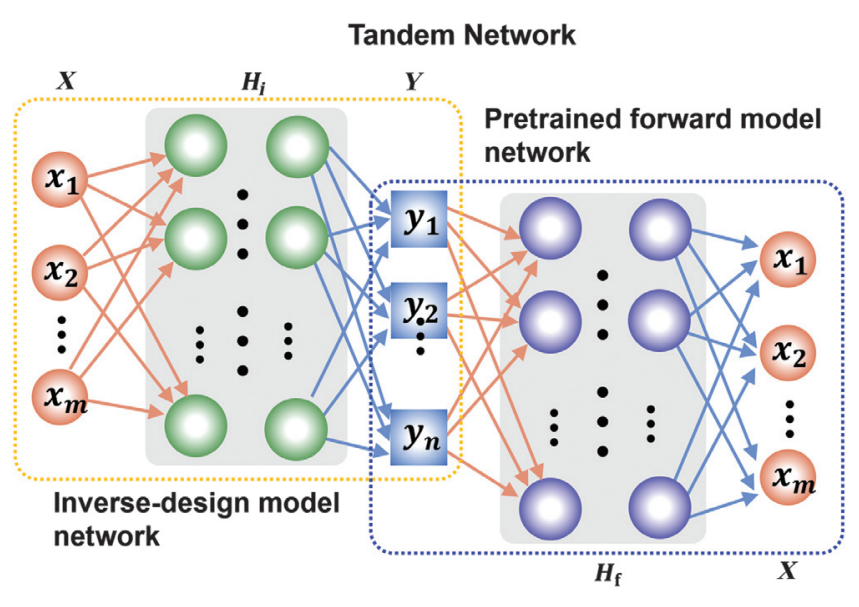

Fig. 2 The architecture of the TN model, which consists of an inverse-design network connected to a pretrained forward model network. $X$ represents the input and output, which is the transmission spectra data in our case, and $Y$ represents the output in the middle layer which is the structural parameters here.

produce a prespecified design. Instead, it is free to infer any design that results in the desired forward behavior.

In our case, for simplicity, we specify three structural parameters of the nanobars to learn: width $w$, length $L$, and the offset distance $x_{0}$. It is worth mentioning that the machine-learning approach can be extended to predict nanostructures with more parameters or even different types of parameters such as material properties and material losses. By randomly specifying them, we first use the rigorous coupled-wave analysis (RCWA) $)^{76}$ to generate 25,000 training examples, in which we obtain the transmission spectra of the metasurfaces covering a wavelength range of 1400 to $1600 \mathrm{~nm}$. Here, the refractive index of silicon in this wavelength range is nearly constant; thus in our simulation, we keep the refractive index of silicon as 3.6 to expedite the training data generation process. RCWA is a frequency-domain modal method based on the decomposition of the periodic structure and the pseudoperiodic solution of Maxwell's equations in terms of their Fourier expansions. ${ }^{77}$ It has been widely used for modeling light responses from periodic optical structures due to its fast convergence and accurate far-field calculations. ${ }^{78}$ It is quite suitable for modeling the electromagnetic responses of metasurfaces and generating massive training data, especially when considering the inverse design based on neural networks. ${ }^{46,51}$ It is worth mentioning that, for multilayer structures, the transfer matrix method can be one more choice. ${ }^{79}$

The forward model network is designed to have four fully connected layers with each layer having 400-600-400-200 dimensions, respectively. We set the learning parameter batch size as 256 , and use a learning rate of 0.001 and decay of $1 \times 10^{-6}$. We first train the forward model network and evaluate it to see how well it can predict the given transmission spectra. Figure 3(a) shows the learning curves for training and validation loss. It can be seen that both the training loss and validation loss decrease significantly after 10,000 epochs of training and become $<0.005$ after 30,000 epochs of training. This indicates that the trained network can estimate an appropriate spectrum that is similar to the spectrum calculated analytically. As a test example, we use RCWA to simulate the transmission spectrum for an individual $\mathrm{Si}$ nanobar metasurface with parameters $\left[w, L, x_{0}\right]$ being $[300,700,300] \mathrm{nm}$, as shown by the black dashed curve of Fig. 3(b). Then, we input these structural parameters to the network and predict the corresponding output, which is shown in the blue curve of Fig. 3(b). As can be seen, our forward network can predict the transmission spectrum from our metasurface accurately. We have also tested our forward network by specifying different structural parameter sets corresponding to different transmission spectra (see Fig. S4 in the Supplemental Material), which all verify the effectiveness of our forward network.

Next, we train the inverse-design model network by fixing the weights in the pretrained forward model network. Since the forward model network is differentiable, we can train the inverse-design model network with a loss placed after the forward model network. As mentioned above, this will overcome the issue of nonuniqueness in the inverse spectrum of electromagnetic waves, as the design by the neural network is not required to be identical to the design parameters that produced the training samples, but the spectrum inferred by the forward model network must match the target spectrum. The loss can be further lowered when the generated design and the real design have similar responses after training. The inverse-design model network has five fully connected layers with 600, 600, 400, 200, 200 dimensions, respectively. For the inverse design of our metasurfaces, the transmission spectrum is considered the input of the TN. The design parameters are predicted from the intermediate layer of the whole network. The training process is shown in Fig. 3(c). As can be seen, using the TN approach, the learning of inverse design has converged effectively. We then test our inverse-design network using a Fano formula to define the transmission spectrum: ${ }^{80,81}$

$F(\omega)=A_{0}+F_{0} \frac{\left[q+2\left(\omega-\omega_{0}\right) / \Gamma\right]^{2}}{1+\left[2\left(\omega-\omega_{0}\right) / \Gamma\right]^{2}}$,

where $\omega_{0}$ and $\Gamma$ are the resonance frequency and linewidth, respectively. $A_{0}$ and $F_{0}$ are constant factors and are fixed at 1 in the rest of this paper. $q$ is a dimensionless factor that describes the ratio between the resonant and nonresonant transition amplitudes in the spectrum. Here, our target is an FR with a peak value of $100 \%$ and dip value of $0 \%$. Therefore, the resonance frequency and linewidth of the transmission resonances can be obtained via

$T(\omega)=\frac{F(\omega)-F_{\min }}{F_{\max }-F_{\min }}$.

We first design a target FR at $\lambda_{0}=1500 \mathrm{~nm}$, with resonance linewidth $\Delta \lambda=5 \mathrm{~nm}$ and $q=0.5$, as shown by the black dashed curve of Fig. 3(d). Here, we input the resonance frequency linewidth $\Gamma$ by the defined resonance wavelength linewidth $\Delta \lambda$ by $\Gamma=\Delta \lambda \omega_{0} / \lambda_{0}$. We then use the network to predict the structural parameters $\left[w, L, x_{0}\right]$ of the required metasurface as $[316,580,189] \mathrm{nm}$. The transmission spectrum of the predicted metasurface is shown in the dashed red curve of Fig. 3(d). It matches well with the desired Fano-shape curve based on Eqs. (1) and (2).

The deep network provides a powerful approach to design nanophotonic structures inversely. Once the training process is finished, the inverse design calculation takes only around $0.05 \mathrm{~s}$ in our case [only using CPU in a normal desktop with 64-bit Operating System: Processor: Intel(R) Core(TM) i7$4770 \mathrm{CPU} @ 3.40 \mathrm{GHz}, \mathrm{RAM}: 16.0 \mathrm{~GB}$ ], which is both 
(a)

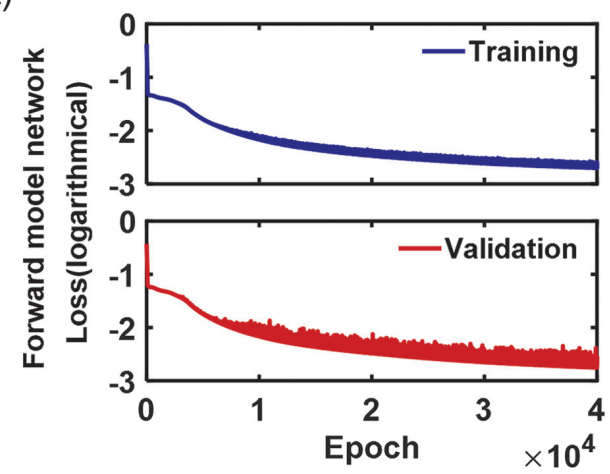

(c)

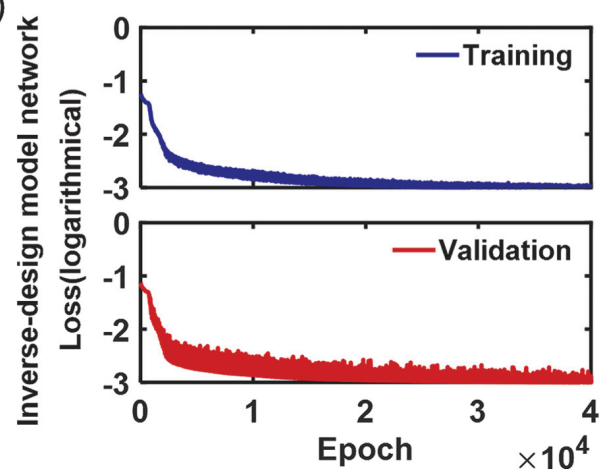

(b)

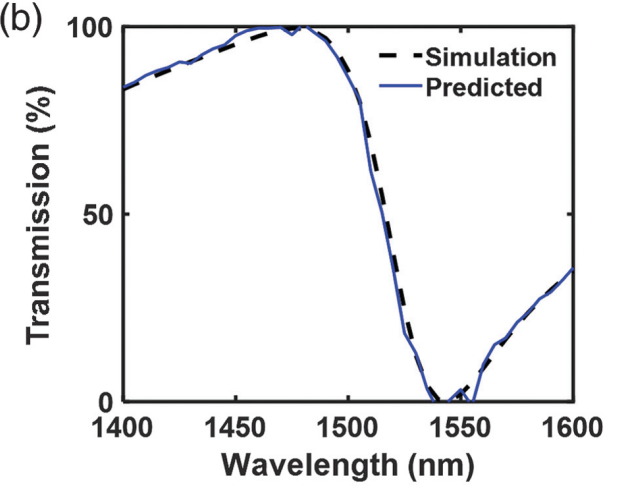

(d)

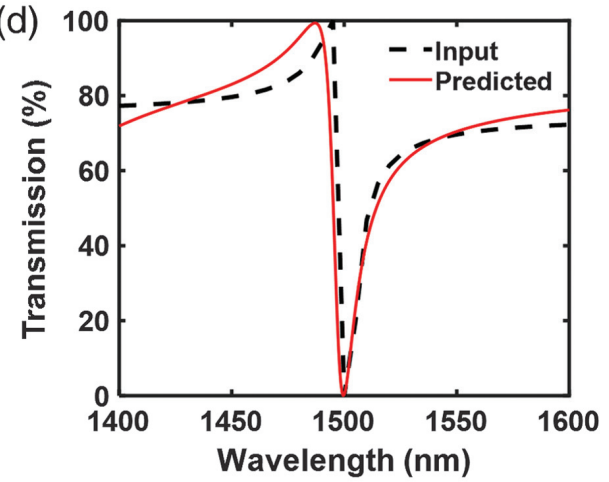

Fig. 3 (a) Evolution of the training loss for the forward model network. (b) Comparison of the NN approximation to the real transmission spectrum. (c) Evolution of the training loss for the inversedesign model network. (d) Comparison of the spectra between the NN approximation and the input based on Eq. (2).

promising and much more effective, as compared with conventional electromagnetic solvers. For nanophotonics applications, as mentioned above, it is a requirement to obtain scalable metasurfaces with controllable characteristics. In the following, using the TN approach, we further test the inverse design of FRs with different spectral positions, linewidths, or amplitudes. According to Eqs. (1) and (2), we first specify target FRs at $\lambda_{0}=1450,1500$, and $1550 \mathrm{~nm}$, respectively. Subsequently, we keep the linewidth $\Delta \lambda=15 \mathrm{~nm}$ and $q=0.8$, as shown in the black dashed curve of Figs. 4(a)-4(c). We then use the trained neural network to predict the structural parameters of the metasurfaces that can provide such spectra. The transmission spectra of the designed metasurfaces are shown in the red curves of Figs. 4(a)-4(c). They match and satisfy the design goal well. By varying the value of parameter $q$ or the linewidth of the resonance $\Delta \lambda$, the neural network can easily predict the metasurface design for the required FR with different characteristics, as shown in Figs. 4(d)-4(i). It provides a powerful method to control the near-field and electric energy confinement at the nanoscale (see Fig. S5 in the Supplemental Material).

So far, based on the deep-learning approach, we have optimized the design to obtain high- $Q$ resonances via altering various parameters, including the linewidth, spectral position, and amplitude, simultaneously. Such a high- $Q$ resonance can significantly facilitate nanostructures to enhance light-matter interactions for various applications such as nonlinear optics and optomechanics. As an example, here, we investigate the THG from three designed metasurfaces with resonances at 1450, 1500, and 1550 nm, as shown in Figs. 4(a)-4(c), respectively.
Here, we have taken into account that the spectral full-width-athalf-maximum of our experimentally used laser is around $15 \mathrm{~nm}$ for the wavelength range 1400 to $1600 \mathrm{~nm}$. Thus, we consider the resonances with this linewidth to maximize the nonlinear signal generation.

The fabrication is done via the standard electrolithography, similar to our previous work, ${ }^{15}$ in which nanostructures are fabricated out of amorphous silicon on a glass substrate. The scanning electron microscope (SEM) image of one fabricated metasurface sample with a designed resonance at $1500 \mathrm{~nm}$ is shown in Fig. 5(a). The measured dimensions of the fabricated metasurfaces $\left[w, L, x_{0}\right]$ are $[269,805,188] \mathrm{nm}$ (resonant at $1450 \mathrm{~nm}$ ), $[290,765,184] \mathrm{nm}$ (resonant at $1500 \mathrm{~nm}$ ), and $[310,780,184] \mathrm{nm}$ (resonant at $1550 \mathrm{~nm}$ ) (see Fig. S6 in the Supplemental Material). While the experimental and theoretical $x_{0}$ are identical, controlled by the lithography software, there are slight differences in the values of the fabricated $w$ and $L$ from the theoretical targeting values, as shown in Figs. 4(a)-4(c), due to fabrication imperfections. Such discrepancies, together with a possible minor inhomogeneity of the experimentally deposited silicon film, slightly affect the experimentally fabricated samples. It is worth noting that the lattice perturbations at the array's edge can also break the periodic boundary conditions leading to scattering of light in all directions into the free space, which weakens the total transmission or total reflection of the FR.

We first measured the linear transmission spectra of the three metasurfaces under plane wave normal incidence with the electric field polarized along the $y$ axis, as shown in Fig. 5(b). Pronounced asymmetric FRs were observed around 
(a)

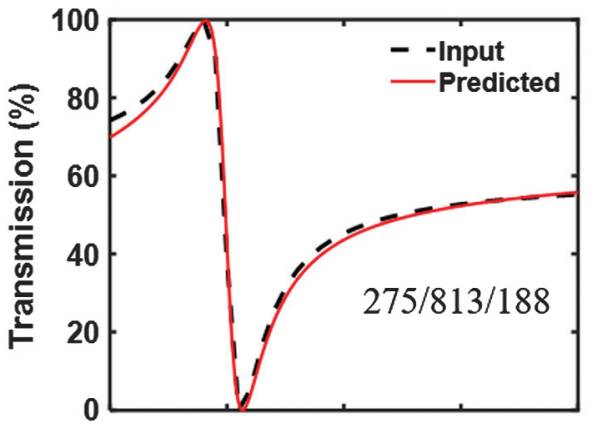

(d)

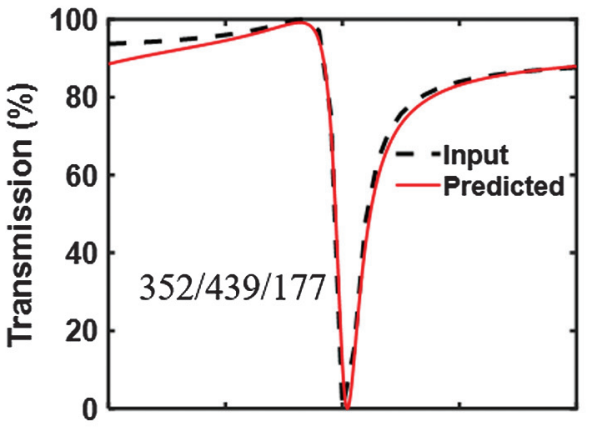

(g)

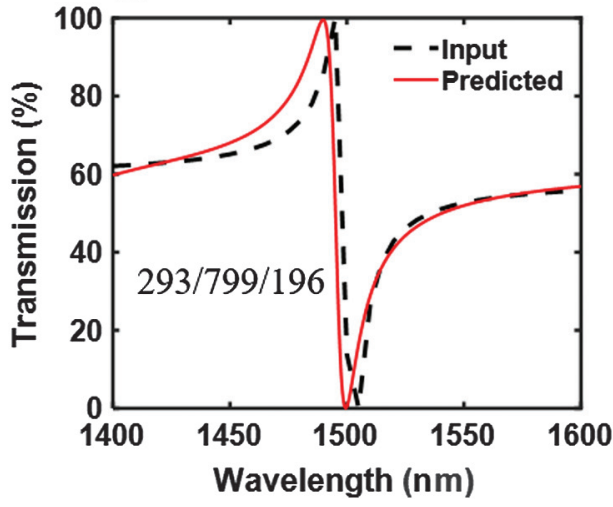

(b)

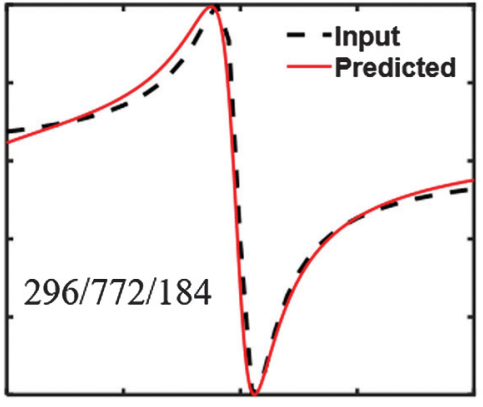

(e)

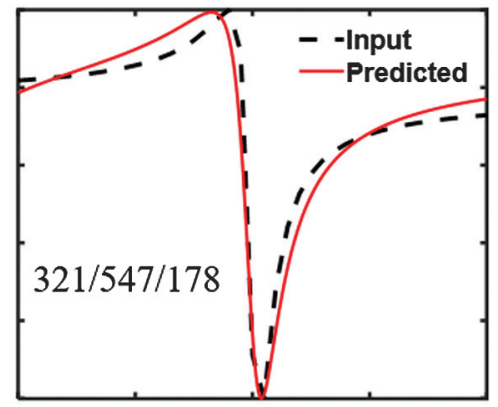

(h)

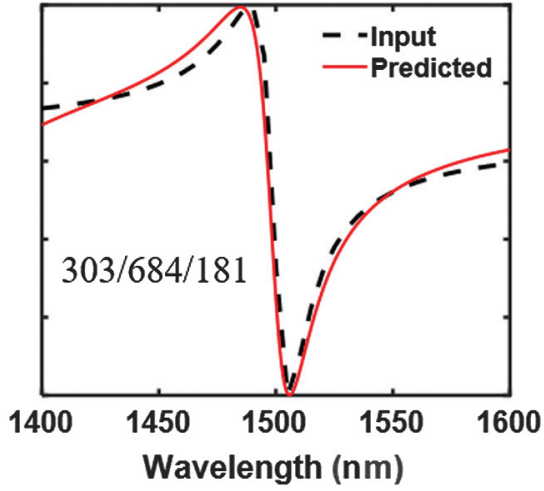

(c)

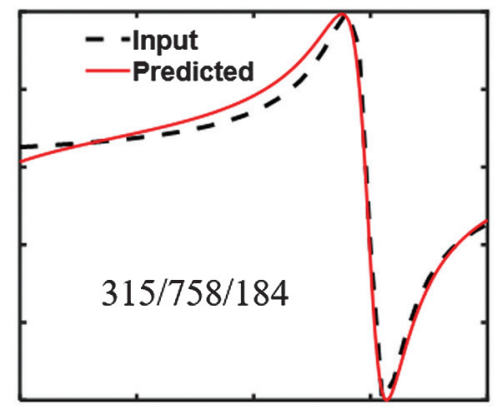

(f)

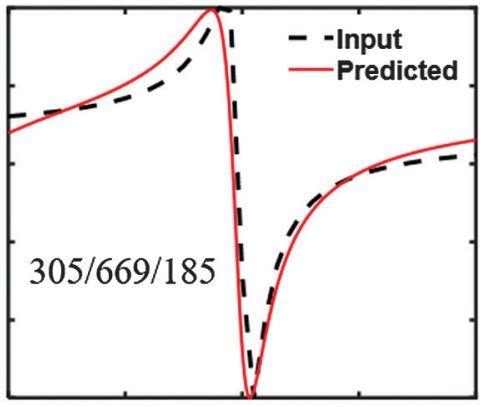

(i)

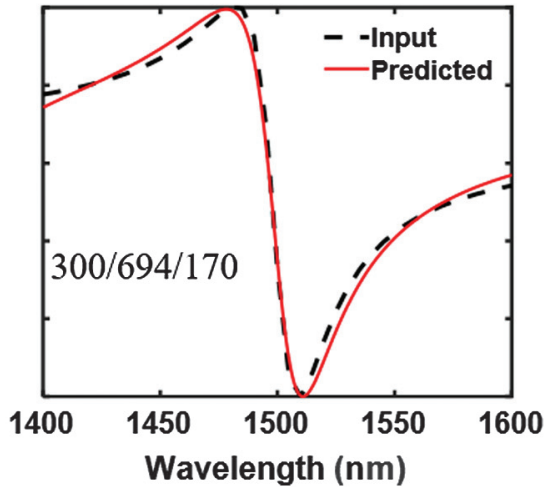

Fig. 4 Inverse design of $\mathrm{Si}$ nanobar metasurfaces with Fano-shape transmission spectra. (a)-(c) $\lambda_{0}=1450,1500$, and $1550 \mathrm{~nm}$, respectively. $\Delta \lambda=15 \mathrm{~nm}, q=0.8$. (d)-(f) $\lambda_{0}=1500 \mathrm{~nm}$, $\Delta \lambda=10 \mathrm{~nm}, q=0.3,0.5$, and 0.7 , respectively. (g)-(i) $\lambda_{0}=1500 \mathrm{~nm}, \Delta \lambda=5,15$, and $25 \mathrm{~nm}$, respectively, $q=0.7$.

the desired spectral positions. Subsequently, we performed the TH spectroscopy measurement. A femtosecond laser beam with 200 fs pulse width and $80 \mathrm{MHz}$ repetition rate was focused by an aspheric lens with a focal length of $5 \mathrm{~cm}$ to a beam waist of $20 \mu \mathrm{m}$. The pump polarization was adjusted along the $y$ axis to excite the designed TD BIC state and was tuned ranging from 1400 to $1600 \mathrm{~nm}$, with maximum mean power in the sample plane up to around $66 \mathrm{~mW}$, leading to a maximum peak intensity value of around $0.66 \mathrm{GW} / \mathrm{cm}^{2}$. An objective with a numerical aperture (NA) NA $=0.7$ was used to collect the transmitted TH emission power in the forward direction (see Fig. S7 in Section V. Experimental setup for nonlinear measurements of the Supplemental Material). The experimentally measured TH signals from the three designed metasurfaces are shown in Fig. 5(c). As can be seen, the TH signals are significantly enhanced around the resonances, while no THG enhancement is observed when the laser beam is polarized along the $x$ axis (see Fig. S8 in the Supplemental Material). By comparison, we observed 400-fold enhancement of the TH signal at the resonance position under the $y$-polarized pump as compared with the case of the $x$-polarized pump (Fig. S8 in the Supplemental Material). Similarly, by performing the nonlinear multipolar analysis, the TH signal is dominated by the TD excitation with small portions of MQ and EQ excitations, which exhibit the same $C_{2}$ symmetry (Fig. S9 in the Supplemental Material). This further leads to a stronger $\mathrm{TH}$ emission in the first-order diffraction compared with the zero-order diffraction due to the absence of coupling to these modes and the normal outgoing waves (see Fig. S9 in the Supplemental Material).

Another advantage of high- $Q$ resonances is that the particular electric field distributions can also facilitate the optomechanical vibration process. ${ }^{82-86}$ In other words, by designing a high- $Q$ 
(a)
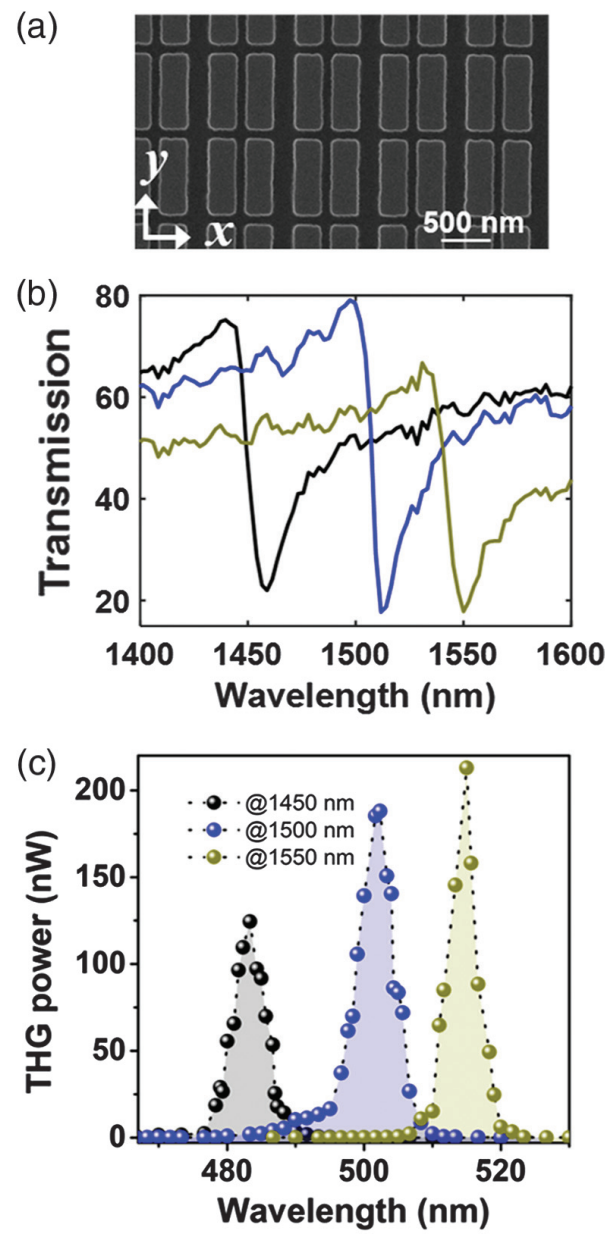

Fig. 5 (a) SEM image of the fabricated sample with designed resonance at $1500 \mathrm{~nm}$. (b) Experimentally measured linear spectra. (c) Experimentally measured THG spectra of the samples.

resonance through machine learning, one can engineer the vibrational modes of nanoparticles based on the optomechanical processes, too. Here, by considering the designed metasurface shown in Fig. 2(d), we numerically investigate the optomechanical vibrations and mechanical mode excitations. The mechanical vibration is modeled as

$$
\begin{aligned}
\rho \frac{\partial^{2} u(r, t)}{\partial t^{2}}= & \nabla \cdot\left[\left(1+\beta \frac{\partial}{\partial t}\right)\left\{C: \frac{[\nabla u(r, t)]^{T}+\nabla u(r, t)}{2}\right\}\right] \\
& +F(r, t),
\end{aligned}
$$

where $u$ is the displacement field characterizing the mechanical vibration and the constants $\rho, C$, and $\beta$ represent mass density, stiffness tensor, and decay time of the silicon material, respectively. $F(r, t)$ is the driving force induced by the electromagnetic field here. Low-loss $\mathrm{Si}$ nanostructures can have a significantly high laser damage threshold: $\sim 400 \mathrm{GW} / \mathrm{cm}^{2}$ or $\sim 100 \mathrm{~mJ} / \mathrm{cm}^{2}$ at 250 fs light pump, and $\sim 1000 \mathrm{GW} / \mathrm{cm}^{2}$ or $\sim 100 \mathrm{~mJ} / \mathrm{cm}^{2}$ at 100 fs light pump. ${ }^{11,87,88}$ Here, the optical pump in our analysis is a single $y$-polarized laser pulse at the wavelength of $1500 \mathrm{~nm}$ with a pulse duration $200 \mathrm{fs}$, peak intensity $I_{0}=50 \mathrm{GW} / \mathrm{cm}^{2}$, and at time $t_{0}=0 \mathrm{~s}$. Here, we numerically calculate the effect of optical force on the mechanical vibration of our nanostructures using COMSOL Multiphysics. The optical force is determined by the time-averaged Maxwell stress tensor: ${ }^{89}$

$$
\begin{aligned}
T_{\alpha \beta}= & \epsilon\left(E_{\alpha}^{*} E_{\beta}+E_{\alpha} E_{\beta}^{*}-\delta_{\alpha \beta}|\mathbf{E}|^{2}\right) \\
& +\mu\left(H_{\alpha}^{*} H_{\beta}+H_{\alpha} H_{\beta}^{*}-\delta_{\alpha \beta}|\mathbf{H}|^{2}\right),
\end{aligned}
$$

where $\delta_{\alpha \beta}=1$ when $\alpha=\beta$ and $\delta_{\alpha \beta}=0$ otherwise. The optical force in the $\alpha$ direction is then derived from the induced optical near-field profiles as

$F_{\alpha}=\int\left(\nabla \cdot T_{\alpha \beta}\right)_{\alpha} \mathrm{d} V$

Due to the symmetric distributions of optical fields in these two nanobars shown in Figs. 1(d) and 6(a), the corresponding vibration process for them is similar and has the same absolute values. Subsequently, we analyze the vibrations by measuring the changes in the width and length along the center of right nanobar in the unit cell [see Fig. 6(a)]. The vibration displacements along the $x$ and $y$ directions, i.e., $D_{x}$ and $D_{y}$, respectively, are shown in Fig. 6(b). To reveal the excitation of acoustic modes under optical pump, we further calculate the spectral density of the optomechanical vibrations. The corresponding spectral density is computed by the Fourier transfer analysis of this time-dependent vibrational amplitude and is plotted in Fig. 6(c), which shows that the coherent phonon oscillation frequency for this nanobar is around $12 \mathrm{GHz}$ for $D_{x}$ and $9.5 \mathrm{GHz}$ for $D_{y}$. For comparison, Figs. 6(d)-6(f) show the corresponding optomechanical vibrations when the pump is polarized along the $x$ axis. The amplitude of optomechanical vibration is around 100 times stronger from the metasurface under $y$-polarized pump incidence, which corresponds to the excitation of the designed TD BIC state. It is worth noting that the optomechanical response and the excitation of acoustic modes are dependent on the dimensions of the nanostructures. Therefore, by geometric tuning of the nanostructures, efficient excitation of different acoustic modes at other frequencies can be achieved.

To demonstrate the importance of high- $Q$ resonance on acoustic modes, we further calculate the spectral densities for different pump wavelengths under $y$-polarized pump incidence, as shown in the three-dimensional (3-D) map in Fig. 7. As can be seen, a significant peak with the frequency of $12 \mathrm{GHz}$ for $D_{x}$ ( $9.5 \mathrm{GHz}$ for $D_{y}$ ) appears for the wavelength at $1500 \mathrm{~nm}$ due to the excitation of the TD BIC state at the optical pump. The excitation strength of the acoustic mode decreases dramatically when the optical pump is away from the TD BIC state. We then estimate the feedback of the mechanical vibration on the resonant optical response. Based on the transient vibration shown in Fig. 6(b), by assuming deformation around 50-pm displacement in the $x$ direction or $25-\mathrm{pm}$ displacement in the $y$ direction, the maximum sensitivity of the scattering response in such metasurfaces is around $0.055 \% \mathrm{pm}^{-1}$ (see Fig. S10 in the Supplemental Material). Utilizing the high-quality TD BIC state, up to $4.5 \%$ modulation of the transmission near the resonance can be expected when using a pulse laser with peak intensity $50 \mathrm{GW} / \mathrm{cm}^{2}$ through the radiation force on the silicon nanostructures. These results suggest new opportunities for optomechanical applications such as light modulation and nanosensing with nanostructures. 
(a)
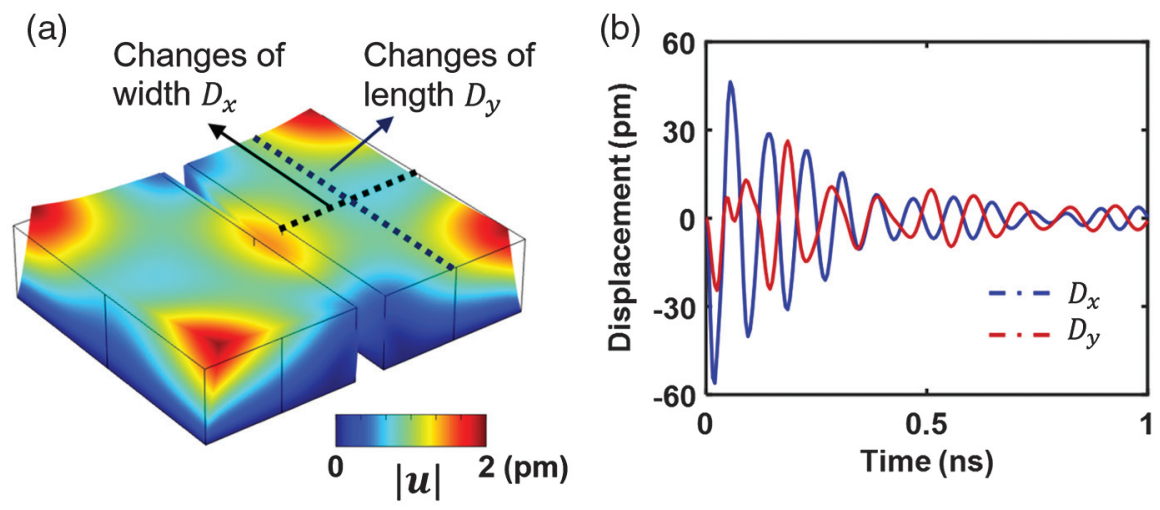

(d)

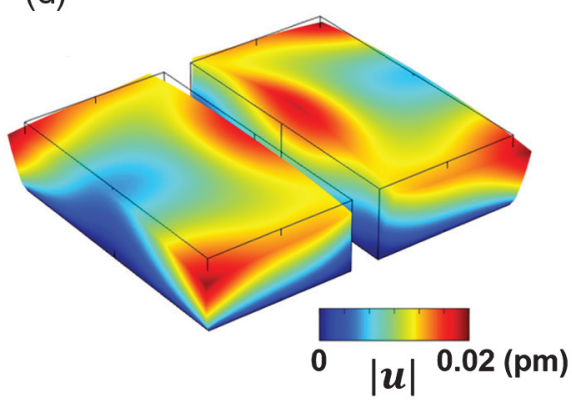

(e)

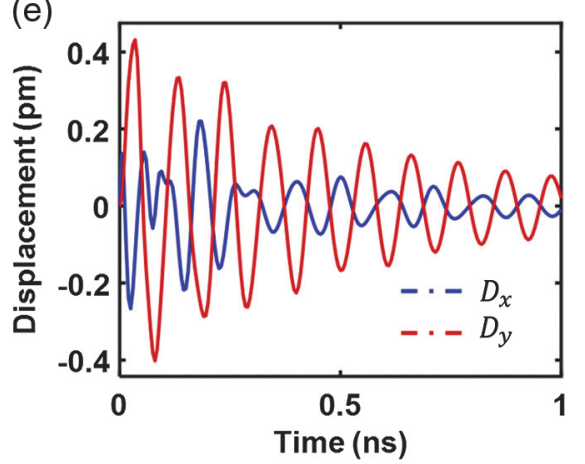

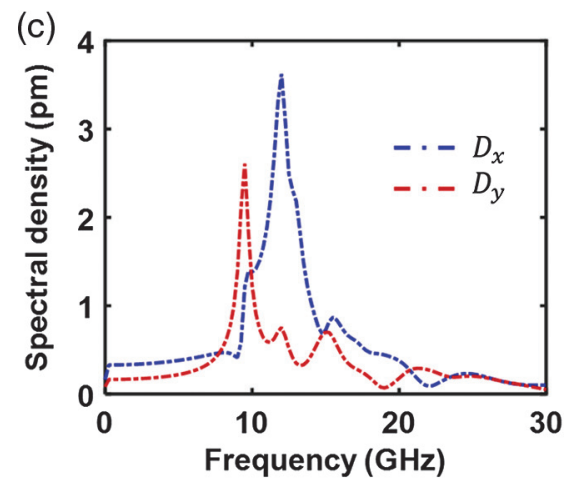

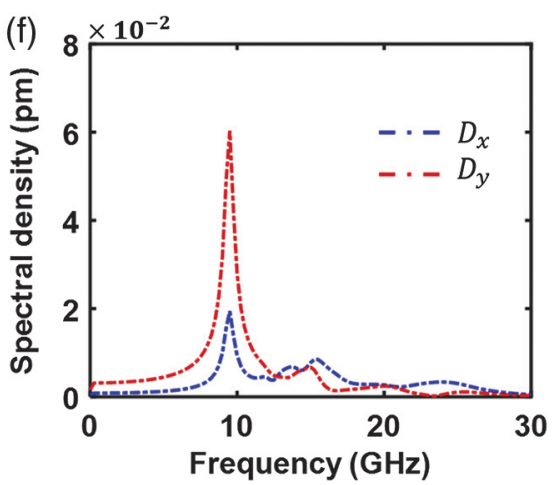

Fig. 6 (a)-(c) Optomechanic vibration under the $y$-polarized pump. (a) Displacement of the nanobars after $1 \mathrm{~ns}$. (b) The transient displacement $D_{x}$ and $D_{y}$. (c) Spectral densities of displacement $D_{x}$ and $D_{y}$. (d)-(f) Optomechanical vibration under the $x$-polarized pump. (d) Displacement of the nanobars after $1 \mathrm{~ns}$. (e) The transient displacement $D_{x}$ and $D_{y}$. (f) Spectral densities of displacement $D_{x}$ and $D_{y}$.
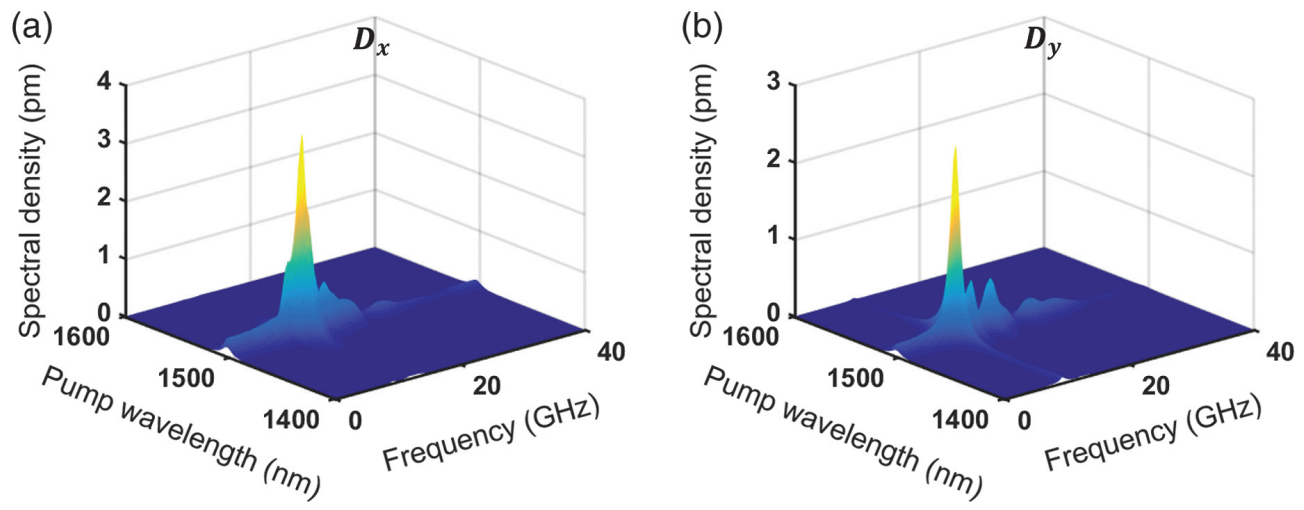

Fig. 7 The spectral density of $D$ in the (a) $x$ and (b) $y$ directions for different laser pump wavelengths.

\section{Conclusion}

Applying a machine-learning approach, we have demonstrated the inverse design of high-quality FR metasurfaces composed of two nanobars with scalable characteristics including the spectral position, line width, and amplitude of the transmission. The FR is originated from the TD-BIC state, featuring a strong nearfield enhancement and intense electric energy localized inside the nanobars. We further employ these metasurfaces to simultaneously enhance photon-photon and photon-phonon interactions and achieve up to 400-fold THG enhancement and up to 100-fold enhancement of the amplitude of optomechanical vibrations. Our proposed scalable metasurfaces suggest new opportunities to control and enhance light-matter interactions, showing promising applications for realizing optoacoustic nonlinear metasurfaces. 


\section{Acknowledgments}

We are grateful to Andrey Sukhorukov, Yue Sun, and Camille Diffine for fruitful discussions. The authors acknowledge the funding support provided by the Australian Research Council (ARC). The work of A.E.M. was supported by UNSW Scientia Fellowship and ARC Discovery Project (DP170103778). M.R. sincerely appreciates funding from ARC Discovery Early Career Research Fellowship (DE170100250). D.S. acknowledges financial support from the Russian Foundation for Basic Research (Grants Nos. 1802-00381 and 19-02-00261) and the Australian Research Council (DE19010043). The authors appreciate the use of the Australian National Fabrication Facility (ANFF)-the ACT Node.

\section{References}

1. D. Neshev and I. Aharonovich, "Optical metasurfaces: new generation building blocks for multi-functional optics," Light Sci. Appl. 7, 58 (2018).

2. C. U. Hail et al., "Optical metasurfaces: evolving from passive to adaptive," Adv. Opt. Mater. 7(14), 1801786 (2019).

3. S. Chang, X. Guo, and X. Ni, "Optical metasurfaces: progress and applications," Annu. Rev. Mater. Res. 48, 279-302 (2018).

4. A. I. Kuznetsov et al., "Optically resonant dielectric nanostructures," Science 354, aag2472 (2016).

5. M. Rahmani et al., "Reversible thermal tuning of all-dielectric metasurfaces," Adv. Funct. Mater. 27(31), 1700580 (2017).

6. A. E. Miroshnichenko, S. Flach, and Y. S. Kivshar, "Fano resonances in nanoscale structures," Rev. Mod. Phys. 82(3), 2257 (2010).

7. B. Luk'yanchuk et al., "The Fano resonance in plasmonic nanostructures and metamaterials," Nat. Mater. 9, 707-715 (2010).

8. V. V. Khardikov, E. O. Iarko, and S. L. Prosvirnin, "A giant red shift and enhancement of the light confinement in a planar array of dielectric bars," J. Opt. 14(3), 035103 (2012).

9. M. Rahmani, B. Luk'yanchuk, and M. Hong, "Fano resonance in novel plasmonic nanostructures," Laser Photonics Rev. 7(3), 329349 (2013).

10. M. Gupta and R. Singh, "Toroidal versus Fano resonances in high Q planar THz metamaterials," Adv. Opt. Mater. 4(12), 2119-2125 (2016).

11. Y. Yang et al., "Nonlinear Fano-resonant dielectric metasurfaces," Nano Lett. 15, 7388-7393 (2015).

12. V. R. Tuz et al., "High-quality trapped modes in all-dielectric metamaterials," Opt. Express 26(3), 2905-2916 (2018).

13. S. Campione et al., "Broken symmetry dielectric resonators for high quality factor Fano metasurfaces," ACS Photonics 3(12), 2362-2367 (2016).

14. K. Z. Kamali et al., "Reversible image contrast manipulation with thermally tunable dielectric metasurfaces," Small 15(15), 1805142 (2019).

15. L. Xu et al., "Dynamic nonlinear image tuning through magnetic dipole quasi-BIC ultrathin resonators," Adv. Sci. 6, 1802119 (2019).

16. A. Jain et al., "Electric and magnetic response in dielectric dark states for low loss subwavelength optical meta atoms," Adv. Opt. Mater. 3(10), 1431-1438 (2015).

17. F. Hao et al., "Tunability of subradiant dipolar and Fano-type plasmon resonances in metallic ring/disk cavities: implications for nanoscale optical sensing," ACS Nano 3(3), 643-652 (2009).

18. N. Liu et al., "Three-dimensional plasmon rulers," Science 332(6036), 1407-1410 (2011).

19. F. Hao et al., "Symmetry breaking in plasmonic nanocavities: subradiant LSPR sensing and a tunable Fano resonance," Nano Lett. 8(11), 3983-3988 (2008).

20. K. Bao, N. A. Mirin, and P. Nordlander, "Fano resonances in planar silver nanosphere clusters," Appl. Phys. A 100(2), 333-339 (2010).
21. N. J. Halas et al., "Plasmons in strongly coupled metallic nanostructures," Chem. Rev. 111(6), 3913-3961 (2011).

22. M. Hentschel et al., "Transition from isolated to collective modes in plasmonic oligomers," Nano Lett. 10(7), 2721-2726 (2010).

23. M. Rahmani et al., "Subgroup decomposition of plasmonic resonances in hybrid oligomers: modeling the resonance lineshape," Nano Lett. 12(4), 2101-2106 (2012).

24. C. W. Hsu et al., "Bound states in the continuum," Nat. Rev. Mater. 1(9), 16048 (2016).

25. L. Carletti et al., "Giant nonlinear response at the nanoscale driven by bound states in the continuum," Phys. Rev. Lett. 121(3), 033903 (2018).

26. K. Koshelev et al., "Asymmetric metasurfaces with high-Q resonances governed by bound states in the continuum," Phys. Rev. Lett. 121(19), 193903 (2018).

27. M. Liu and D.-Y. Choi, "Extreme Huygens' metasurfaces based on quasi-bound states in the continuum," Nano Lett. 18(12), 80628069 (2018).

28. Y. He et al., "Toroidal dipole bound states in the continuum," Phys. Rev. B 98(16), 161112 (2018).

29. K. Koshelev et al., "Nonlinear metasurfaces governed by bound states in the continuum," ACS Photonics 6(7), 16391644 (2019).

30. X. Luo et al., "Excitation of high Q toroidal dipole resonance in an alldielectric metasurface," Opt. Mater. Express 10(2), 358-368 (2020).

31. L. Carletti et al., "High-harmonic generation at the nanoscale boosted by bound states in the continuum," Phys. Rev. Res. 1(2), 023016 (2019).

32. H. K. Gandhi et al., "Gain-loss engineering of bound states in the continuum for enhanced nonlinear response in dielectric nanocavities," Opt. Express 28(3), 3009-3016 (2020).

33. R. F. Ndangali and S. V. Shabanov, "Electromagnetic bound states in the radiation continuum for periodic double arrays of subwavelength dielectric cylinders," J. Math. Phys. 51(10), 102901 (2010).

34. V. F. Gili et al., "Monolithic AlGaAs second-harmonic nanoantennas," Opt. Express 24, 15965-15971 (2016).

35. S. Liu et al., "Resonantly enhanced second-harmonic generation using III-V semiconductor all-dielectric metasurfaces," Nano Lett. 16, 5426-5432 (2016).

36. R. Camacho-Morales et al., "Nonlinear generation of vector beams from AlGaAs nanoantennas," Nano Lett. 16, 7191-7197 (2016).

37. G. Grinblat et al., "Efficient third harmonic generation and nonlinear subwavelength imaging at a higher-order anapole mode in a single germanium nanodisk," ACS Nano 11, 953-960 (2017).

38. G. Grinblat et al., "Enhanced third harmonic generation in single germanium nanodisks excited at the anapole mode," Nano Lett. 16, 4635-4640 (2016).

39. M. R. Shcherbakov et al., "Ultrafast all-optical switching with magnetic resonances in nonlinear dielectric nanostructures," Nano Lett. 15(10), 6985-6990 (2015).

40. E. V. Melik-Gaykazyan et al., "Third-harmonic generation from Mie-type resonances of isolated all-dielectric nanoparticles," Philos. Trans. R. Soc. A 375, 20160281 (2017).

41. E. V. Melik-Gaykazyan et al., "Selective third-harmonic generation by structured light in Mie-resonant nanoparticles," ACS Photonics 5(3), 728-733 (2017).

42. A. E. Miroshnichenko et al., "Nonradiating anapole modes in dielectric nanoparticles," Nat. Commun. 6, 8069 (2015).

43. L. Xu et al., "Boosting third-harmonic generation by a mirror-enhanced anapole resonator," Light Sci. Appl. 7, 44 (2018).

44. L. Carletti et al., "Enhanced second-harmonic generation from magnetic resonance in AlGaAs nanoantennas," Opt. Express 23, 26544-26550 (2015).

45. S. Molesky et al., "Inverse design in nanophotonics," Nat. Photonics 12(11), 659-670 (2018).

46. D. Liu et al., "Training deep neural networks for the inverse design of nanophotonic structures," ACS Photonics 5(4), 1365-1369 (2018). 
47. A. Mirzaei et al., "Superscattering of light optimized by a genetic algorithm," Appl. Phys. Lett. 105(1), 011109 (2014).

48. C. C. Nadell et al., "Deep learning for accelerated all-dielectric metasurface design," Opt. Express 27(20), 27523-27535 (2019).

49. Y. Kiarashinejad, S. Abdollahramezani, and A. Adibi, "Deep learning approach based on dimensionality reduction for designing electromagnetic nanostructures," npj Comput. Mater. 6(1), 12 (2020).

50. W. Ma, F. Cheng, and Y. Liu, "Deep-learning-enabled on-demand design of chiral metamaterials," ACS Nano 12(6), 6326-6334 (2018).

51. K. Yao, R. Unni, and Y. Zheng, "Intelligent nanophotonics: merging photonics and artificial intelligence at the nanoscale," Nanophotonics 8(3), 339-366 (2019).

52. Z. Liu et al., "Integrated nanophotonic wavelength router based on an intelligent algorithm," Optica 6(10), 1367-1373 (2019).

53. L. Gao et al., "A bidirectional deep neural network for accurate silicon color design," Adv. Mater. 31(51), 1905467 (2019).

54. Q. Zhang et al., "Artificial neural networks enabled by nanophotonics," Light Sci. Appl. 8, 42 (2019).

55. J. Jiang and J. A. Fan, "Simulator-based training of generative neural networks for the inverse design of metasurfaces," Nanophotonics (2019).

56. S. So, J. Mun, and J. Rho, "Simultaneous inverse design of materials and structures via deep learning: demonstration of dipole resonance engineering using core-shell nanoparticles," ACS Appl. Mater. Interfaces 11(27), 24264-24268 (2019).

57. J. Jiang and J. A. Fan, "Global optimization of dielectric metasurfaces using a physics-driven neural network," Nano Lett. 19(8), 5366-5372 (2019)

58. P. R. Wiecha et al., "Pushing the limits of optical information storage using deep learning," Nat. Nanotechnol. 14(3), 237-244 (2019).

59. A. Y. Piggott et al., "Inverse design and demonstration of a compact and broadband on-chip wavelength demultiplexer," Nat. Photonics 9(6), 374-377 (2015).

60. T. Asano and S. Noda, "Optimization of photonic crystal nanocavities based on deep learning," Opt. Express 26(25), 32704-32717 (2018).

61. G. Carleo et al., "Machine learning and the physical sciences," Rev. Mod. Phys. 91(4), 045002 (2019).

62. G. Carleo and M. Troyer, "Solving the quantum many-body problem with artificial neural networks," Science 355(6325), 602-606 (2017).

63. J. A. Hertz, Introduction to the Theory of Neural Computation, CRC Press, Boca Raton, Florida (2018).

64. K. Hornik et al., "Multilayer feedforward networks are universal approximators," Neural Networks 2(5), 359-366 (1989).

65. H. Aouani et al., "Third-harmonic-upconversion enhancement from a single semiconductor nanoparticle coupled to a plasmonic antenna," Nat. Nanotechnol. 9, 290-294 (2014).

66. J. Lee et al., "Giant nonlinear response from plasmonic metasurfaces coupled to intersubband transitions," Nature 511(7507), 6569 (2014).

67. M. Merklein et al., "Enhancing and inhibiting stimulated Brillouin scattering in photonic integrated circuits," Nat. Commun. 6, 6396 (2015).

68. R. Pant et al., "On-chip stimulated Brillouin scattering," Opt. Express 19(9), 8285-8290 (2011).

69. R. Van Laer et al., "Interaction between light and highly confined hypersound in a silicon photonic nanowire," Nat. Photonics 9 , 199-203 (2015).

70. G. Grinblat et al., "High-efficiency second harmonic generation from a single hybrid $\mathrm{ZnO}$ nanowire/Au plasmonic nanooligomer," Nano Lett. 14(11), 6660-6665 (2014).

71. F. Della Picca et al., "Tailored hypersound generation in single plasmonic nanoantennas," Nano Lett. 16(2), 1428-1434 (2016).

72. H. Aouani et al., "Unveiling the origin of third harmonic generation in hybrid ITO-plasmonic crystals," Adv. Opt. Mater. 3(8), 1059-1065 (2015).
73. Y. S. Chen et al., "Silica-coated gold nanorods as photoacoustic signal nanoamplifiers," Nano Lett. 11(2), 348-354 (2011).

74. L. Moreaux et al., "Coherent scattering in multi-harmonic light microscopy,” Biophys. J. 80, 1568-1574 (2001).

75. F. Chollet, "Keras: deep learning library for Theano and TensorFlow," GitHub Repository (2015).

76. M. G. Moharam et al., "Formulation for stable and efficient implementation of the rigorous coupled-wave analysis of binary gratings," J. Opt. Soc. Am. A 12(5), 1068-1076 (1995).

77. J. P. Hugonin and P. Lalanne, "Reticolo software for grating analysis," Institute of Optics Graduates School, Orsay, France (2005).

78. A. Arbabi et al., "Dielectric metasurfaces for complete control of phase and polarization with subwavelength spatial resolution and high transmission," Nat. Nanotechnol. 10, 937-943 (2015).

79. J. Peurifoy et al., "Nanophotonic particle simulation and inverse design using artificial neural networks," Sci. $A d v$. 4(6), eaar4206 (2018).

80. I. S. Maksymov and A. E. Miroshnichenko, "Active control over nanofocusing with nanorod plasmonic antennas," Opt. Express 19(7), 5888-5894 (2011).

81. M. Galli et al., "Light scattering and Fano resonances in high-Q photonic crystal nanocavities," Appl. Phys. Lett. 94, 071101 (2009).

82. F. Medeghini et al., "Controlling the quality factor of a single acoustic nanoresonator by tuning its morphology," Nano Lett. 18(8), 5159-5166 (2018).

83. Y. Gan and Z. Sun, "Crystal structure dependence of the breathing vibration of individual gold nanodisks induced by the ultrafast laser," Appl. Opt. 58(1), 213-218 (2019).

84. Y. Sun et al., "Opto-mechanical interactions in nanoparticles with magnetic light," in Opt. InfoBase Conf. Papers (2016).

85. A. Ivinskaya et al., "Optomechanical manipulation with hyperbolic metasurfaces," ACS Photonics 5(11), 4371-4377 (2018).

86. C. Yi et al., "Polycrystallinity of lithographically fabricated plasmonic nanostructures dominates their acoustic vibrational damping," Nano Lett. 18(6), 3494-3501 (2018).

87. E. Shamonina, World Scientific Handbook of Metamaterials and Plasmonics, World Scientific, Oxford (2017).

88. S. Makarov et al., "Tuning of magnetic optical response in a dielectric nanoparticle by ultrafast photoexcitation of dense electron-hole plasma," Nano Lett. 15(9), 6187-6192 (2015).

89. J. D. Jackson, Classical Electrodynamics, John Wiley \& Sons, New York (1999).

Lei $\mathrm{Xu}$ is a research fellow at School of Engineering and Information Technology, University of New South Wales, Canberra, Australia. He obtained his PhD in optics from Nankai University (Tianjin, China) in 2014, followed by postdocs at Nankai University and Australian National University. His main research interests are linear and nonlinear nanophotonics, metasurfaces, and optoelectronic metadevices.

Mohsen Rahmani received his $\mathrm{PhD}$ from the National University of Singapore in 2013. He is a senior member of IEEE and his research interests span over nonlinear nanophotonics and optical metasurfaces. $\mathrm{He}$ is a recipient of several prestigious recognitions, including the Australian Eureka Prize (2018), and Early Career Medal from the International Union of Pure and Applied Physics (2017). His contribution to this paper has been a part of his project for ARC DECRA on optoacoustic metasurfaces.

Stephen Gould is a professor of computer science at the ANU. He is a former Microsoft Faculty Fellow and Amazon Principal Research Scientist, and currently a director of the Australian Centre for Robotic Vision. He received his $\mathrm{BSc}$ and $\mathrm{BE}$ degrees from the University of Sydney in 1994 and 1996, respectively. He received his MSEE from Stanford in 1998. After several years in industry he returned to academia 


\section{Xu et al.: Enhanced light-matter interactions in dielectric nanostructures via machine.}

and earned his PhD from Stanford in 2010. He has broad interests in computer vision and machine learning and optimization.

Dragomir N. Neshev is a professor in physics at the Australian National University (ANU) and the director of the Australian Research Council Centre of Excellence for Transformative Meta-Optical Systems. He received his PhD from Sofia University, Bulgaria, in 1999. Since then he has worked in the field of optics at several research centres and joint the ANU in 2002. His activities span over several branches of optics, including periodic photonic structures, singular optics, plasmonics, and optical metasurfaces.
Andrey E. Miroshnichenko obtained his $\mathrm{PhD}$ from the Max-Planck Institute for Physics of Complex Systems in Dresden, Germany, in 2003. In 2004, he moved to Australian National University. During that time, he made fundamentally important contributions to the field of photonic crystals and bringing the concept of the Fano resonances to nanophotonics. In 2017, he moved to the University of New South Wales Canberra. The topics of his research are nanophotonics, nonlinear and quantum optics, and resonant interaction of light with nanoclusters, including optical nanoantennas and metamaterials.

Biographies of the other authors are not available. 